\title{
Study of Soft X-ray Emission from Sahand as Filippov Type Plasma Focus Using Different Cathode Type
}

\author{
M.A. Mohammadi ${ }^{a, b, *}$, M. Manouchehrizadeh ${ }^{a}$ And R.S. RAWAT ${ }^{c}$ \\ ${ }^{a}$ Department of Atomic and Molecular Physics, Faculty of Physics, University of Tabriz, Tabriz, Iran \\ ${ }^{b}$ Research Center of Astrophysics and Applied Physics, University of Tabriz, Tabriz, Iran \\ ${ }^{c}$ Natural Sciences and Science Education, National Institute of Education, \\ Nanyang Technological University, Singapore
}

(Received November 13, 2019; in final form March 12, 2020)

\begin{abstract}
To the best knowledge of the authors, so far, the detailed experimental effect of the cathode type on the $\mathrm{X}$-ray yield in the Filippov type plasma focus devices has not been documented in literatures. Our results show that changing the cathode, affects significantly on the efficiency of X-ray production. Therefore, the main aim of the current study is to provide experimental data showing that the open-squirrel cage cathode is the best choice regarding the X-ray yield for typical Filippov type plasma focus at least among a few different types of cathodes that widely have been used. The effect of three different cathode types (i.e., open-squirrel cage, close-squirrel cage, and cylindrical) on the pinch current and neon soft X-ray yield is investigated experimentally in a plasma focus device. The results confirm that the pinch current depends on the cathode type and in open-squirrel cage cathode it is higher than two other cases. The results also confirm that a large fraction of the maximum current is transferred to the plasma column. Experimental results also show that the time difference between pinch and maximum currents with open-squirrel cage cathode is less than two other cathodes. Using two channel diode X-ray spectrometer, the effect of cathode type on the neon soft X-ray emission at constant pressure (0.5 Torr) and different voltages $(10-15 \mathrm{kV})$ is investigated. In the open-squirrel cage cathode with increasing voltage, the average neon soft X-ray yield increases approximately linearly whereas for close-squirrel cage and cylindrical cathodes the neon soft X-ray yield has a maximum (at $12 \mathrm{kV}$ for cylindrical and $13 \mathrm{kV}$ for squirrel cage cathode). The maximum neon soft X-ray yield for the open-squirrel cage cathode is $21.9 \pm 3.4 \mathrm{~J}$ at $15 \mathrm{kV}$ and for the cylindrical cathode $16.4 \pm 1.2 \mathrm{~J}$ at $12 \mathrm{kV}$ and for close-squirrel cage cathode $15.9 \pm 3.4 \mathrm{~J}$ at $13 \mathrm{kV}$.
\end{abstract}

DOI: 10.12693/APhysPolA.137.1089

PACS/topics: plasma focus, Filippov type, cathode, neon soft X-ray

\section{Introduction}

Dense plasma focus (DPF) has for many years been used to produce pulsed hot and dense pinch plasma. The DPF was independently developed in the early 1960s by Mather [1] (USA) and by Filippov [2] (ex-USSR) in two configurations. The difference between these two devices was in their geometrical configuration. The pinch plasma produced in the DPF device has high temperature $(\approx 1 \mathrm{keV})$ and density $\left(\approx 1025-1026 \mathrm{~m}^{-3}\right)$. Historically, the DPF has been established as an alternative fusion facility due to the intense bursts of neutrons it produces when operated with deuterium as a working gas [3-6] studied.

In the DPF, when the high voltage pulse is applied between the electrodes, an azimuthal symmetric electrical discharge will be initiated. The discharge is preceded by an electrical breakdown phase which generates an initial plasma configuration through which the discharge current can flow. When the sliding discharge reaches the end of the insulator, then it connects both electrodes. Due to the $\boldsymbol{J} \times \boldsymbol{B}$ force the current is lifted off in an inverse pinch manner. After some time $(50 \approx 500 \mathrm{~ns})$

*corresponding author; e-mail: mohammadidorbash@yahoo.com the conductance of the sliding discharge becomes high enough and the discharge converts into a current sheath (CS), in fact, a double-layer structure consisting of the ionization front and the magnetic piston [7-11]. The CS formed at the end of the breakdown phase is accelerated by its own $\boldsymbol{J} \times \boldsymbol{B}$ force towards the open end of the inner electrode. This phase is called axial phase. At the end of the axial phase, the CS sweeps around the end of the inner electrode (anode) and finally collapses due to the radially inward $\boldsymbol{J} \times \boldsymbol{B}$ force.

The hot and dense pinch plasma column of the DPF devices produces highly energetic ions [12-16], relativistic electrons [17] and soft/hard X-rays [18-24]. In the DPF device, the highly energetic ions have been used for material processing such as an ion implantation and thin film depositions [25-27]. Similarly, many experimental studies on X-ray emission from DPF were oriented to various applications such as X-ray lithography, X-ray radiography and micromachining [28-34]. For applications like lithography and micromachining soft X-rays (SXR) have been used. In these applications of SXR, it is important to detect and measure the SXR yield. Typically, silicon PIN diodes, together with adequate filters, have been used for the detection and estimation of SXR yields [11, 28, 29, 35]. The BPX65 type PIN diode has been commonly used. BPX65 has inherent fast rise time and high quantum efficiency. The typical parameters of this PIN diode are listed in Table I. 
Typical parameters of BPX65 PIN diode.

TABLE I

\begin{tabular}{l|c}
\hline \hline \multicolumn{1}{c|}{ Parameter } & Value \\
\hline radiant sensitive area & $1 \mathrm{~mm}^{2}$ \\
intrinsic Si wafer thickness & $10 \mu \mathrm{m}$ \\
dead layer thickness & $0.5 \mu \mathrm{m}$ \\
rise time (typical) $(900 \mathrm{~nm})$ & $0.5 \mathrm{~ns}$ \\
dark current & $<5 \mathrm{nA}($ nom. $1 \mathrm{nA})$
\end{tabular}

There are many parameters of DPF devices, such as gas type, working pressure, charging voltage, system inductance and type (shape and material) of anode and cathode, which affect the SXR yield [18, 21, 36]. Previously, there was no documented clear explanation, either theoretical or experimental, that why a particular type of cathode should be used. In this paper, the effect of cathode type (i.e., open-squirrel cage, close-squirrel cage, and cylindrical) at various capacitor bank charging voltages on the SXR yield, maximum current, the current flowing into the plasma current sheath (pinch current) and the time difference between the pinch and maximum currents with neon as a working gas have been studied.

\section{Experimental setup and diagnostic}

In this investigation, the Fillipov-type Sahand plasma focus was used. The energy necessary for realizing the discharge and for the plasma production is stored in the capacitor store consisting of 24 capacitors. The capacity of each condenser is $12 \mu \mathrm{F}$, maximal charging voltage is $25 \mathrm{kV}$. The total power supply source capacity is $288 \mu \mathrm{F}$. The maximal stored energy is $90 \mathrm{~kJ}$. The peak discharge current is about 1.1 MA and the inductance is $40 \mathrm{nH}$. The Sahand plasma focus device has four main parts. The first part is discharge chamber which is a stainless steel cylinder with the diameter of $76 \mathrm{~cm}$ and the height of $26 \mathrm{~cm}$. The second main part is a $50 \mathrm{~cm}$ diameter copper disc that acts as the anode. The anode is connected to the spark gap via 24 copper rods. The third part is a ceramic cylinder with the diameter of $48 \mathrm{~cm}$ and the height of $11 \mathrm{~cm}$ that acts as an insulator and encloses the anode disc as shown in Fig. 1 [8]. The fourth part is the cathode. In this study, we used three different cathode types (i.e., open-squirrel cage, close-squirrel cage, and cylindrical). The schematic views of Sahand with different cathode types are shown in Fig. 1. The open-squirrel cage cathode is the conventional squirrel cage configuration used in plasma focus device consisting of 24 open-ended brass rods located at $37 \mathrm{~cm}$ from anode axis (Fig. 1a). The height of each rod is $19 \mathrm{~cm}$. In the cylindrical cathode, we considered the chamber of plasma focus as our cathode, i.e., cathode rods were removed (Fig. 1b). Finally, the close-squirrel cage cathode has the same conventional open-squirrel cage cathode but with a superimposed plate on top of the rods as shown in Fig. 1c. The characteristics of all cathodes are listed in Table II.

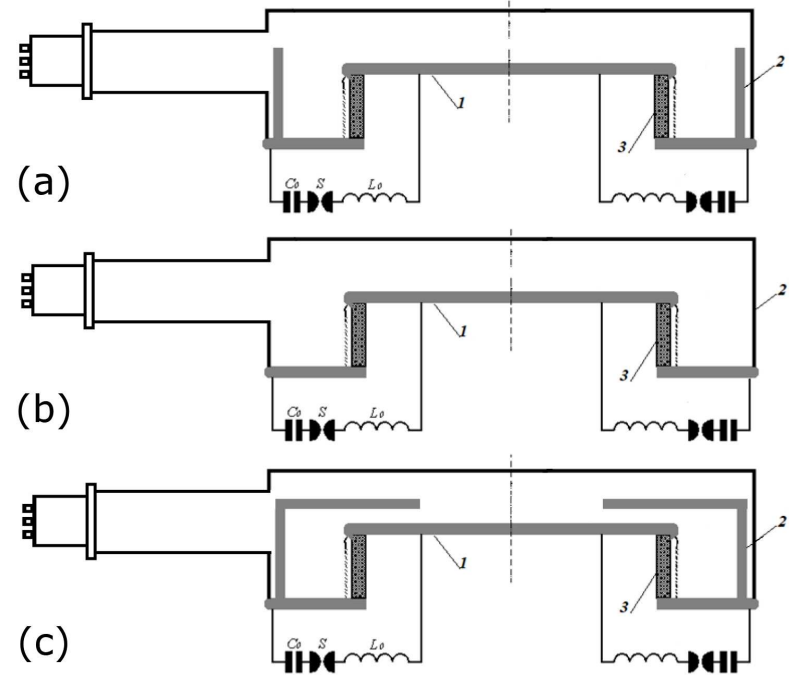

Fig. 1. Schematic view of Sahand plasma focus with three types of cathodes: (a) open-squirrel cage (conventional type), (b) cylindrical, and (c) close-squirrel cage (conventional squirrel cage cathode rods covered with a plate at the top); with (1) anode, (2) cathode, (3) insulator.

Cathodes characteristics.

TABLE II

\begin{tabular}{l|c|c|c|c}
\hline \hline \multicolumn{1}{c|}{ Electrode type } & $\begin{array}{c}\text { Radius } \\
{[\mathrm{mm}]}\end{array}$ & $\begin{array}{c}\text { Height } \\
{[\mathrm{mm}]}\end{array}$ & $\begin{array}{c}\text { Thickness } \\
{[\mathrm{mm}]}\end{array}$ & $\begin{array}{c}\text { Number } \\
\text { of rods }\end{array}$ \\
\hline cylindrical cathode & 380 & 260 & - & - \\
\hline $\begin{array}{l}\text { open-squirrel } \\
\text { cage cathode }\end{array}$ & 370 & 190 & - & 24 \\
\hline $\begin{array}{l}\text { superimposed plate } \\
\text { on the rods } \\
\text { for close-squirrel } \\
\text { cage cathode }\end{array}$ & 370 & - & 10 & 24 \\
\hline
\end{tabular}

Previous studies considered only two configurations open-squirrel cage and cylindrical chamber cathode. Verma's study [37] was performed on the neutron yield with two different cathodes in a miniature plasma focus device. Bruzzone's study [38] was carried out on temporal evolution of the inductance of the plasma-electrodes system. In another study, a close-squirrel cage cathode has been studied for the Filippov-type plasma focus [39]. They studied SXR emission with photon energies of $<1 \mathrm{keV}$ using PF. Therefore, so far, the detailed experimental effect of the cathode type on the X-ray yield and pinch current behavior in the Filippov type plasma focus devices have not been documented in literatures. It is of interest for us to see what would be the effect of three different cathodes types on the SXR emission. As well as in this study, we have studied the behavior of pinch current for each cathode in Sahand as a relatively large plasma focus device. In this paper, for all of the cathode types, the filling neon gas pressure is constant and the initial energy of the device is variable. 
Typical parameters of Rogowski coil.

\begin{tabular}{l|c|l|c}
\hline \hline \multicolumn{1}{c|}{ Parameter } & Value & \multicolumn{1}{c|}{ Parameter } & Value \\
\hline radius & $42 \mathrm{~cm}$ & wire type & copper \\
number of turns & 2100 & wide diameter & $1.0 \mathrm{~mm}$ \\
diameters & $1.4 \mathrm{~cm}$ & calibration factor & $10^{-4} I_{p}$
\end{tabular}

In this experiment, neon was used as the working gas with the pressure of 0.5 Torr to study the SXR emission. For monitoring of current and current derivative signals, the Rogowski coil was used. The typical parameters of Rogowski coil are shown in Table III. Here $I_{p}$ is the plasma current.

The main diagnostic used for recording X-ray emission is a five channel PIN diode X-ray spectrometer (DXS). The silicon PIN diodes, together with suitable filters, have been employed as pulsed X-ray detectors. The PIN diode's intrinsic fast rise times, high quantum efficiency and excellent stability in intense radiation environments makes it convenient device for detecting pulsed X-rays emitting from plasma focus devices. In particular, the BPX65 type PIN diode has been commonly used. It is known that semiconductor detectors can be utilized in a specific energy range. The lower limit of such domain is determined by absorption in the dead layer, and incomplete absorption determines the upper limit. Lower energy X-rays may be detected provided that the dead layer can be thin. When a semiconductor detector absorbs photon with energy $h \nu, h \nu / \omega$ electron-hole pair is produced. Here $\omega$ is the average energy needed to create an electron-hole pair. The quantum detection efficiency of diode is given by

$$
Q=\frac{h \nu}{\omega} \exp \left(-\mu_{d} t_{d}\right)\left[1-\exp \left(-\mu_{s} t_{s}\right)\right]
$$

Here $t_{d}$ and $t_{s}$ are thicknesses of dead layer and sensitive region, respectively, while $\mu_{d}$ and $\mu_{s}$ are absorption coefficients of dead layer and sensitive region, respectively. In the silicon pin diode, since the mobility of both electrons and holes is high then the response time is very short. This time is an important advantage for measurement of pulsed X-ray sources. The signals from DXS also were used to estimate the total radiated X-ray energy. Appropriate pair of filters signals recorded by the DXS provide valuable information on the time evolution of the X-rays in the DPF and the X-ray yield in specific energy range. The five-channel DXS assembly mainly consists of an array of five windowless silicon PIN diodes (BPX65) in order to exclude special range of photon energy, different X-ray filtration foils covered the detector channels. The DXS is located at one meter distance from pinch zone as shown in Fig. 1. In this investigation, we only used two channels with BPX65 photodiodes covered with (i) $20 \mu \mathrm{m} \mathrm{Al}$, and (ii) $10 \mu \mathrm{m} \mathrm{Al}+125 \mu \mathrm{m}$ Mylar as filters. A Tektronix four-channel digital oscilloscope (200 MHz bandwidth and sample rate of $2 \mathrm{GS} / \mathrm{s}$ ) was used to record SXR, current and current derivative signals. The results were obtained for the average of 15 shots for each charging voltage.

\section{Results and discussion}

In this work, the capacitor bank charging voltage in the range of $10-15 \mathrm{kV}$ is used. The typical current, current derivative and X-ray signals are shown in Fig. 2. A deep dip in current derivative signal, and a simultaneous drop in current signal at about $6-7 \mu \mathrm{s}$ show that the plasma focus device pinched efficiently. Because of the fast variation of plasma inductance of the pinching plasma during the radial compression phase, a negative peak in the current derivative appeared. The FWHM of X-ray signals is about 80 ns. As is clear in Fig. 2, X-ray signals and the current derivative signal are acquired at the same time. This means that the time difference between the formation and destruction of the plasma column is very low. The typical current and current derivative signals of three cathode types (close-squirrel cage, cylindrical, and open-squirrel cage) at the same operating conditions of pressure and charging ( 0.5 Torr and $15 \mathrm{kV})$ are shown in Fig. 3. Figure 3 shows that the formation of the plasma column in the open-squirrel cage cathode is faster than that in the two other cathodes.

The average of the maximum current (the statistical average of maximum current for 15 shots) for three cathodes are shown in Fig. 4. One can see there that with increasing charging voltage the maximum current increases almost linearly. The results of Fig. 4 also show that the maximum current for all voltages by using the open-squirrel cage cathode is more than that of other two cathodes. There was no significant difference in maximum currents for other two cathode types. Considering that all operating conditions are the same except the cathode types, it can be concluded that when using the open-squirrel cage cathode, the inductance is less than the other two cathode types (according Lee's model [40]) as the maximum current $I_{0}$ depends on the inverse square root of external inductance $L_{0}$, namely $I_{0}=V_{0} / \sqrt{L_{0} C_{0}}$.

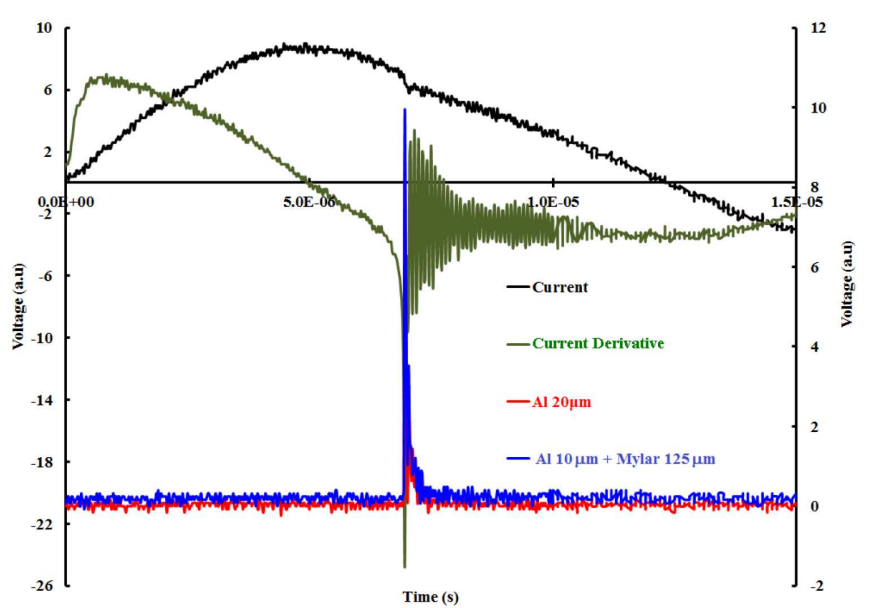

Fig. 2. Typical current, current derivative and X-ray signals. 

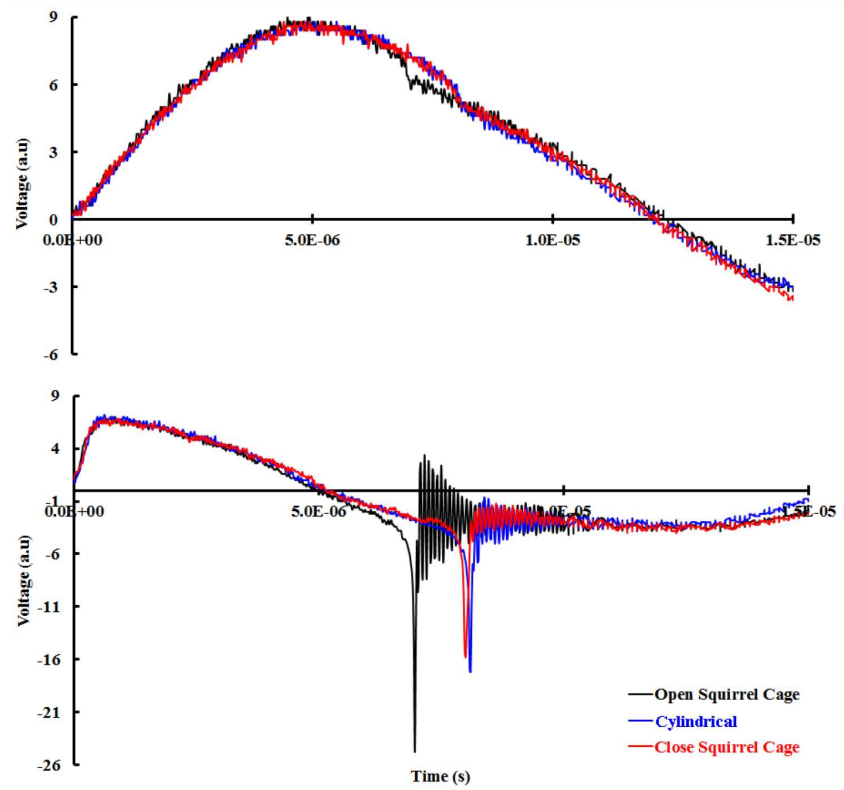

Fig. 3. Typical current and current derivative signal for three types of cathode.

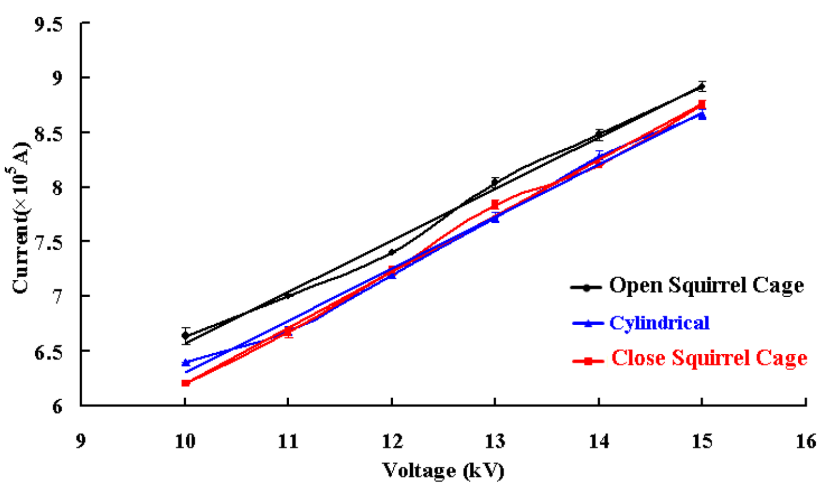

Fig. 4. The variation of maximum current with charging voltage for three cathodes.

In the DPF the cathode's radius changes affect on the current [41]. The variation of average pinch current with working voltage for three different types of cathode is shown in Fig. 5. We found that with increasing voltage from $11 \mathrm{kV}$, the open-squirrel cage cathode has the highest pinch current transferred into the plasma column. After voltage $11 \mathrm{kV}$, when the initial energy increases, the curves of the close-squirrel cage cathode and the cylindrical cathode close together and pinch current for the cylindrical cathode is greater than the close-squirrel cage cathode. However, when the voltage is above $14 \mathrm{kV}$, the pinch current for the close-squirrel cage cathode is greater than that for the cylindrical cathode (see Fig. 5).

The variation of the average time difference between pinch and maximum currents $\left(t_{p}-t_{m}\right)$ with working voltage is shown in Fig. 6. The results show that the time difference between pinch and maximum currents with the

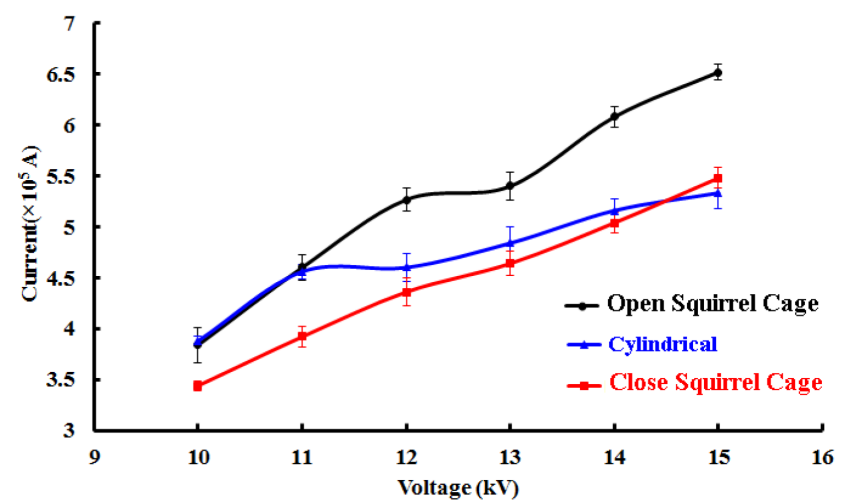

Fig. 5. Pinch current variation with voltage for three cathodes.

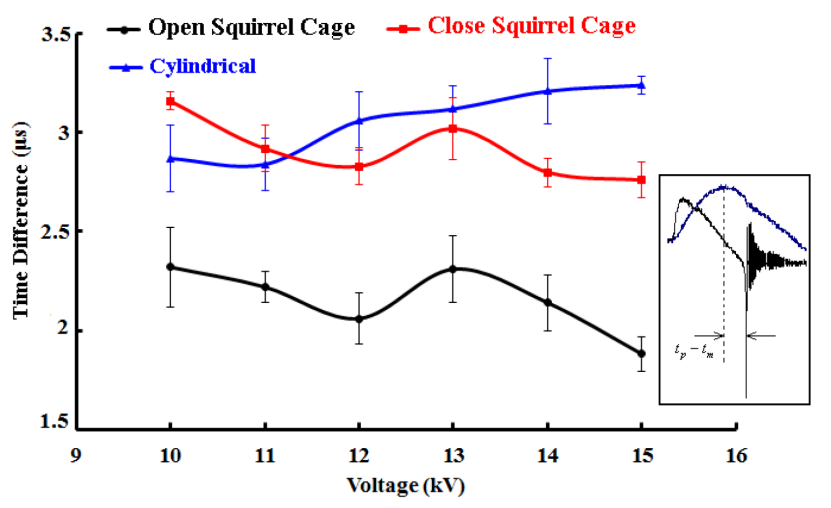

Fig. 6. Variation of time to pinch with voltage for three cathode types.

cylindrical cathode is more than that of the other two cathodes. It may be interesting to note that the change in cathode configuration does affect the current sheath load in the plasma focus device. With using a cylinder as a cathode the current sheath load increases [37]. This means that in this cathode the current sheath velocity is less than other two cathodes.

In Fig. 7 the percentage ratio of pinch current to maximum current with different voltage is shown for three cathode types. For the three different cathode types, the trends of the percentage ratio of pinch current to maximum current with device energy are not similar. The curvature of the close-squirrel cage cathode is smaller than that of the two other cathodes. In the cylindrical cathode, curve trends are increasing, attaining a maximum and then decreasing. Based on results in Fig. 7 we also conclude that with using open-squirrel cage cathode more energy is transferred to the plasma column. In the axial and radial phase, magnetic force is proportional to the current. When the ratio of pinch current to maximum current is greater, then the current percentage flowing into the plasma current sheath increases. This means that with using the open-squirrel cage cathode more energy is transferred into the plasma. 


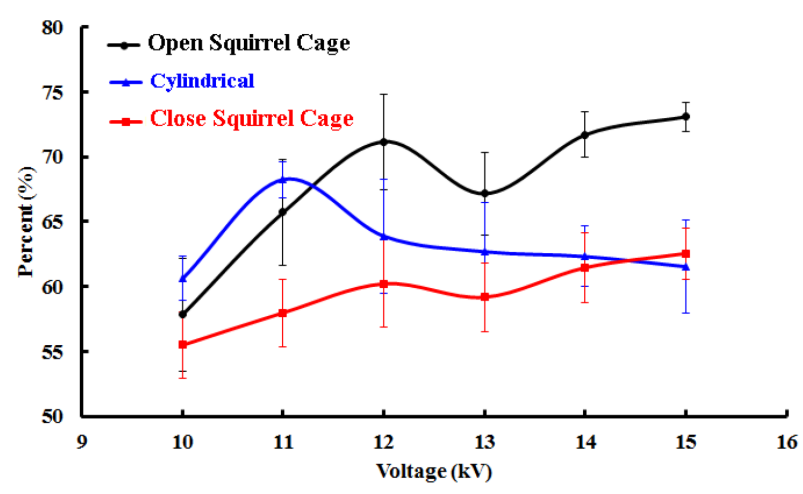

Fig. 7. The percentage ratio of pinch to maximum current with different voltage for three cathodes.

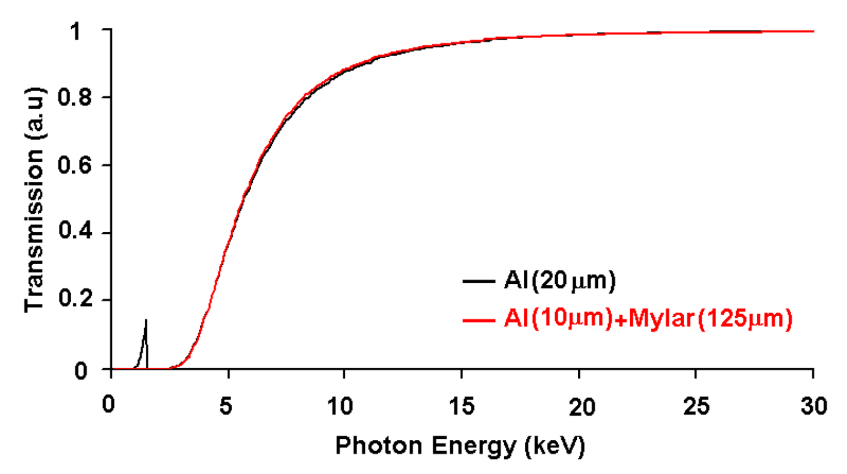

Fig. 8. Transmission curve of $20 \mu \mathrm{m} \mathrm{Al}$ and $10 \mu \mathrm{m} \mathrm{Al}$ plus $125 \mu \mathrm{m}$ Mylar.

From results of Figs. 3-7, we found that for Sahand plasma focus the open-squirrel cage cathode is the best as it makes pinch current to be higher at the most operating voltage which will result in more efficient pinching efficiency. As mentioned in the experimental section, the neon SXR signals were recorded using two channels of DXS comprising of windowless BPX65 photodiodes covered with Ross filter pair of (i) $\mathrm{Al}(20 \mu \mathrm{m})$ and (ii) $\mathrm{Al}(10 \mu \mathrm{m})+\operatorname{Mylar}(125 \mu \mathrm{m})$ as filters. When two channels are covered with $10 \mu \mathrm{m} \mathrm{Al}$ foil, their sensitivities are balanced and normalized by obtaining their signals simultaneously from a plasma focus discharge.

The transmission curves of $\mathrm{Al}(20 \mu \mathrm{m})$ and $\mathrm{Al}(10 \mu \mathrm{m})$ plus Mylar $(125 \mu \mathrm{m})$ are shown in Fig. 8. To measure the neon SXR yield, the spectrum obtained by Liu [11] was employed. In Fig. 8, we found that these filters are appropriate for detecting of neon SXR in the energy range of $900-1550 \mathrm{eV}[11,18]$.

Figure 9 shows the variation of neon SXR yield with charging voltage for three types of cathodes. The results show that with using the cylindrical and closesquirrel cage cathodes the SXR yield is nonlinear and for open-squirrel cage cathode is approximately linear at higher voltages. Maximum yield for cathodes are $16.4 \pm 1.2 \mathrm{~J}$ at $12 \mathrm{kV}$ for cylindrical, $15.9 \pm 3.4 \mathrm{~J}$ at $13 \mathrm{kV}$ for close-squirrel cage, and $21.9 \pm 3.4 \mathrm{~J}$ at $15 \mathrm{kV}$

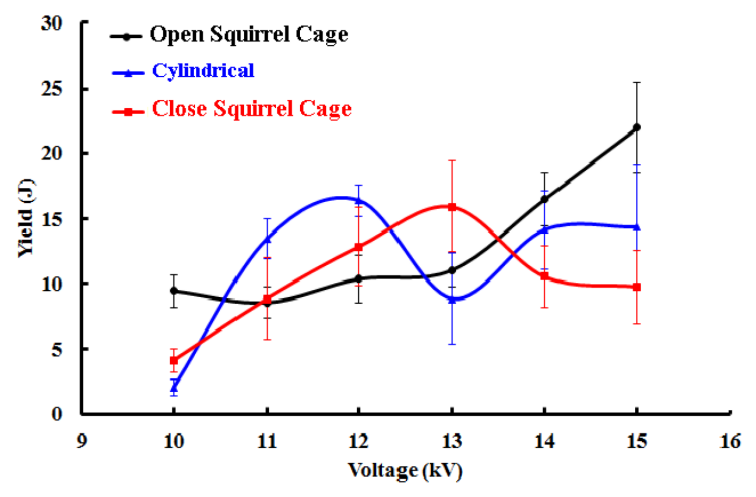

Fig. 9. The variation of neon SXR yield with different voltage for three cathodes.

for open-squirrel cage cathodes. This indicates that the cathode type changes the optimum voltage of SXR yield. From these results, we also find that with using opensquirrel cage cathode we can achieve more SXR yield. In the cases of cylindrical and close-squirrel cage cathodes, outward moving of current sheath reflected back along with impurities from cathode walls. In any case, with the use of open-squirrel cage the space between rods allows mass to pass and so in the inter-electrode space the impurity reduces.

\section{Conclusions}

In the Sahand as a Fillipov type plasma focus, the change on the cathode type is found as an important parameter which affects the current and X-ray emission of the neon filled gas. With using the open-squirrel cage cathode, the pinch current is higher than that of other two cathodes (close-squirrel cage and cylindrical). The Rogowski coil signals show that the time difference between the pinch and maximum currents with opensquirrel cage cathode is less than that of other two cathodes. The results confirm that in the open-squirrel cage cathode, the ratio of pinch current to the maximum current is greater than for other two cathodes. The two channel DXS signals confirm that in the open-squirrel cage cathode with increase of voltage from 11 to $15 \mathrm{kV}$ the average neon SXR increases approximately linearly, whereas for the close-squirrel cage and cylindrical cathode the neon SXR yield is nonlinear. The maximum neon SXR yield for the open-squirrel cage cathode is about $21.9 \pm 3.4 \mathrm{~J}$ at $15 \mathrm{kV}$, and $16.4 \pm 1.2 \mathrm{~J}$ at $12 \mathrm{kV}$ and $15.9 \pm 3.4 \mathrm{~J}$ at $13 \mathrm{kV}$ for the cylindrical cathode and close-squirrel cage cathode, respectively. Finally, experimental results show that the open-squirrel cage is the best cathode for Sahand plasma focus using as a SXR sources.

\section{References}

[1] J.W. Mather, Phys. Fluids 8, 366 (1965).

[2] N. Filippov, T. Filippova, V. Vinogradov, Nucl. Fusion 2, 577 (1962). 
[3] M.J. Bernstein, F. Hai, Phys. Lett. A 31, 3171970 (1970).

[4] A. Bernard, P. Cloth, H. Conrads, A. Coudeville, G. Gourlan, A. Jolas, Ch. Maisonnier, J.P. Rager, Nucl. Instrum. Methods 145, 191 (1977).

[5] P. Silva, J. Moreno, L. Soto, L. Birstein, R.E. Mayer, W. Kies, Appl. Phys. Lett. 83, 3269 (2003).

[6] M.A. Mohammadi, S. Sobhanian, R.S. Rawat, Phys. Lett. A 375, 3002 (2011).

[7] M.A. Mohammadi, S. Sobhanian, M. Ghomeishi, E. Ghareshabani, M. Moslehi-fard, S. Lee, R.S. Rawat, J. Fusion Energy 28, 371 (2009).

[8] M.A. Mohammadi, S. Hedyeh, Plasma Sci. Technol. 17, $353(2015)$

[9] S. Al-Hawat, IEEE Trans. Plasma Sci. 32, 764 (2004).

[10] A.V. Oginov, M.A. Karakin, V.I. Krauz, in: Proc. 15th Int. Conf. on High-Power Particle Beams, St. Petersburg, 2004, p. 746 .

[11] M.H. Liu, Ph.D. Thesis, Nanyang Technological University, Singapore 1996

[12] E. Ghareshabani, M. Mohammadi, J. Fusion Energy 31, 595 (2012).

[13] C. San Wong, P. Choi, W. Seong Leong, J. Singh, Jpn. J. Appl. Phys. 41, 3943 (2002).

[14] H. Kelly, A. Márquez, Plasma Phys. Cont. Fus. 38, 1931 (1996).

[15] H. Kelly, A. Lepone, A. Marquez, IEEE Trans. Plasma Sci. 25, 455 (1997).

[16] H. Bhuyan, H. Chuaqui, M. Favre, I. Mitchell, E. Wyndham, J. Phys. D Appl. Phys. 38, 1164 (2005).

[17] A. Patran, D. Stoenescu, R.S. Rawat, S.V. Springham, T.L. Tan, L.C. Tan, M.S. Rafique, P. Lee, S. Lee, J. Fusion Energy 25, 57 (2006)

[18] M.A. Mohammadi, S. Sobhanian, C.S. Wong, S. Lee, P. Lee, R.S. Rawat, J. Phys. D Appl. Phys. 42, 045203 (2009).

[19] H. Bhuyan, S.R. Mohanty, N.K. Neog, S. Bujarbarua, J. Appl. Phys. 95, 240 (2004).

[20] M.A. Mohammadi, A. Piri, M. Manochehrizadeh, R.S. Rawat, J. Fusion Energy 36, 3002 (2017).

[21] M. Shafiq, S. Hussain, A. Waheed, M. Zakaullah, Plasma Source Sci. Technol. 12, 199 (2003).
[22] M. Zakaullah, K. Alamgir, M. Shafiq, M. Sharif, A. Waheed, IEEE Trans. Plasma Sci. 36, 2089 (2002).

[23] S. Lee, R.S. Rawat, P. Lee, S.H. Saw, J. Appl. Phys. 106, 1 (2009).

[24] S.H. Saw, P.C.K. Lee, R.S. Rawat, S. Lee, IEEE Trans. Plasma Sci. 37, 1276 (2009).

[25] M. Valipour, M.A. Mohammadi, S. Sobhanian, R.S. Rawat, J. Fusion Energy 31, 65 (2012).

[26] S. Karamat, R.S. Rawat, T.L. Lan, P. Lee, S.V. Springham, E. Ghareshbani, R. Chen, H.D. Sun, Appl. Surf. Sci. 257, 1979 (2011).

[27] R.S. Rawat, IEEE Trans. Plasma Sci. 41, 701 (2013).

[28] S. Hussain, S. Ahmad, M.Z. Khan, M. Zakaullah, A. Waheed, J. Fusion Energy 22, 195 (2003).

[29] W. Neff, J. Eberle, R. Holz, R. Lebert, F. Richter, SPIE 1140, 13 (1989).

[30] F. Beg, I. Ross, A. Lorenz, J.F. Worley, A.E. Dangor, M.G. Haines, J. Appl. Phys. 88, 3225 (2000).

[31] V.A. Gribkov, A. Srivastava, P.L.C. Keat, V. Kudryashov, S. Lee, IEEE Trans. Plasma Sci. 30, 1331 (2002).

[32] S. Hussain, M. Shafiq, R. Ahmad, A. Waheed, M. Zakaullah, Plasma Sour. Sci. Technol. 14, 61 (2005).

[33] Y. Kato, S. Be, App. Phys. Lett. 48, 686 (1986).

[34] R.S. Rawat, T. Zhang, G.J. Lim et al., J. Fusion Energy 23, 49 (2004)

[35] D. Wong, A. Patran, T.L. Tan, R.S. Rawat, P. Lee, IEEE Trans. Plasma Sci. 32, 2227 (2004).

[36] S. Lee, S.H. Saw, Plasma Science and Technology for Emerging Economies, Springer, 2017.

[37] R. Verma, R.S. Rawat, P. Lee, S. Lee, S.V. Springham, T.L. Tan, M. Krishnan, Phys. Lett A 373, 2568 (2009).

[38] H. Bruzzone, A. Clausse, M. Barbaglia, H. Acuña, Plasma Phys. Cont. Fus. 54, 1 (2011).

[39] S.A. Dan'ko, K.N. Mitrofanov, V.I. Krauz et al., Plasma Phys. Rep. 41, 882 (2015).

[40] S. Lee, J. Fusion Energy 33, 319 (2014).

[41] S. Lee, A. Serban, IEEE Trans. Plasma Sci. 24, 1101 (1996). 